\title{
Enophthalmos as the Initial Systemic Finding of Undiagnosed Metastatic Breast Carcinoma
}

This article was published in the following Dove Press journal:

International Medical Case Reports Journal

\author{
Jessica El-Khazen Dupuis \\ Michael Marchand (D) \\ Simon Javidi (iD \\ Tuan Quynh Tram Nguyen
}

Department of Ophthalmology, Centre Hospitalier de l'Université de Montréal (CHUM), Montreal, QC H2X 3E4,

Canada
Correspondence: Michael Marchand Department of Ophthalmology, Centre Hospitalier de l'Université de Montréal, I05I Rue Sanguinet, Montréal, QC H2X 3E4, Canada

Tel +I (5I4) 890-8000

Email mic9@sympatico.ca
Purpose: To report on the importance of detecting and investigating non-traumatic enophthalmos, which occurred as the first presenting sign of an undiagnosed metastatic breast carcinoma in two patients with no prior history of neoplasia.

Design: Case series.

Observations: The first case consists of a 74-year-old woman with no significant past medical history, who presented with a non-traumatic enophthalmos and ptosis of her left eye, and horizontal diplopia on right-gaze. Imaging revealed an intraconal lesion of her left orbit, with orbital fat atrophy. Transcutaneous anterior orbitotomy was performed for tumor biopsy, and the histopathology study concluded on a diagnosis of orbital metastasis consistent with infiltrative breast carcinoma. Thorough breast imaging and multiple breast biopsies were not able to localize the primary tumor. The second case consists of a 76-year-old woman, with no prior relevant medical history, who presented for progressive enophthalmos and ptosis of her right eye. Imaging revealed an osteolytic lesion of her right frontal bone, and multiple infiltrative lesions implicating both orbits. A biopsy was performed through a transcutaneous anterior orbitotomy and histopathology study lead to a diagnosis of metastatic lobular breast carcinoma. The primary breast tumor was localized using positron emission tomography, and further biopsy confirmed the diagnosis.

Conclusion: Although uncommon, non-traumatic enophthalmos has a broad differential diagnosis. In some rare instances, it may be the initial presentation of orbital metastases in patients with no prior history of cancer, and in the absence of other systemic symptoms. Clinicians must be thorough when assessing and investigating this clinical entity. A comprehensive eye exam, systemic evaluation, orbit imaging, biopsy and immunohistochemistry analysis are essential to promptly diagnose orbital metastases and plan the appropriate treatment.

Keywords: enophthalmos, breast carcinoma, breast cancer, orbitotomy biopsy

\section{Plain Language Summary}

Metastatic breast cancer can affect the orbits and subsequently result in ocular symptoms such as posterior displacement of the eye which is called enophthalmos. The authors report the cases of two women in whom enophthalmos was the first sign of a previously undiagnosed metastatic breast cancer; these patients had no systemic symptoms at presentation and no prior history of cancer. Although this phenomenon has been described previously, it remains extremely rare for enophthalmos to be the sole feature of a plurimetastatic cancer.

\section{Introduction}

Enophthalmos is a clinical entity defined as a posterior displacement of the ocular globe in relation to the bony orbital margin. ${ }^{1}$ This retrodisplacement can be explained by two 
different pathogenic mechanisms, both resulting in an imbalance between the bony orbit and its contents. ${ }^{1}$ The size of the orbit may be increased, as encountered with trauma, congenital abnormalities, chronic sinusitis with orbital wall destruction, and more. ${ }^{1}$ The second mechanism implies a decrease in the volume of the orbital contents, such as seen in postinflammatory changes within the orbit, or secondary to orbital fat atrophy. ${ }^{1}$ In some rare instances, fat atrophy can occur in the setting of an orbital malignancy, including metastasis. ${ }^{1,2}$

Orbital metastases (OMs) represent a small (1-13\%), but increasing percentage of all orbital neoplasms. ${ }^{1,3}$ Among metastatic orbital tumors, breast carcinoma is considered the most prevalent primary tumor, followed by primary tumors of the lung and prostate. ${ }^{1}$ However, of all possible clinical presentations of OMs, including exophthalmos, diplopia, ocular pain, eyelid swelling and more, enophthalmos is seen in approximately $7-24 \%$ of $\operatorname{cases}^{1,3}$ and almost exclusively with metastatic breast carcinoma. ${ }^{1}$ In only a small percentage of cases $(19 \%)$ will the ophthalmologic symptoms precede the diagnosis of a primary tumor and in even fewer cases (10\%), the primary tumor may remain undetected despite systemic evaluation. ${ }^{3}$ Herein, we report the cases of two women who developed enophthalmos as the first clinical manifestation of a previously unsuspected metastatic breast cancer.

\section{Findings}

Case I

A 74-year-old woman, with no significant prior medical history, presented to our institution for a progressive nontraumatic left eye (OS) enophthalmos and ptosis. When questioned, the patient reported binocular diplopia on right gaze, which had first been noticed three years prior. Her Snellen best corrected visual acuity (BCVA) was 20/30 for the right eye (OD) and 20/40 OS (stable in regard to her baseline BCVA), with an intraocular pressure of $16 \mathrm{~mm} \mathrm{Hg}$ and 13 $\mathrm{mm} \mathrm{Hg}$ for OD and OS, respectively. The left unilateral enophthalmos was confirmed with Hertel exophthalmometer readings of $8 \mathrm{~mm}$ OS, compared to $12 \mathrm{~mm}$ OD (Figure 1). Examination also revealed resistance to globe retropulsion OS, restriction of extra-ocular movements OS ( -2 elevation; -1 abduction; -3 adduction; -2 depression) and left exotropia of 20 prism diopters (PD) on right gaze. There was no eyelid erythema nor induration. Pupillary examination (including evaluation for relative afferent pupillary defect), colour vision (Hardy-Rand-Rittler (HRR) pseudoisochromatic plates test) and dilated fundus exam were within normal limits.

Cerebral computed tomography (CT)-scan and orbital magnetic resonance imaging (MRI) revealed an intraconal lesion sheathing the left optic nerve, and ipsilateral orbital
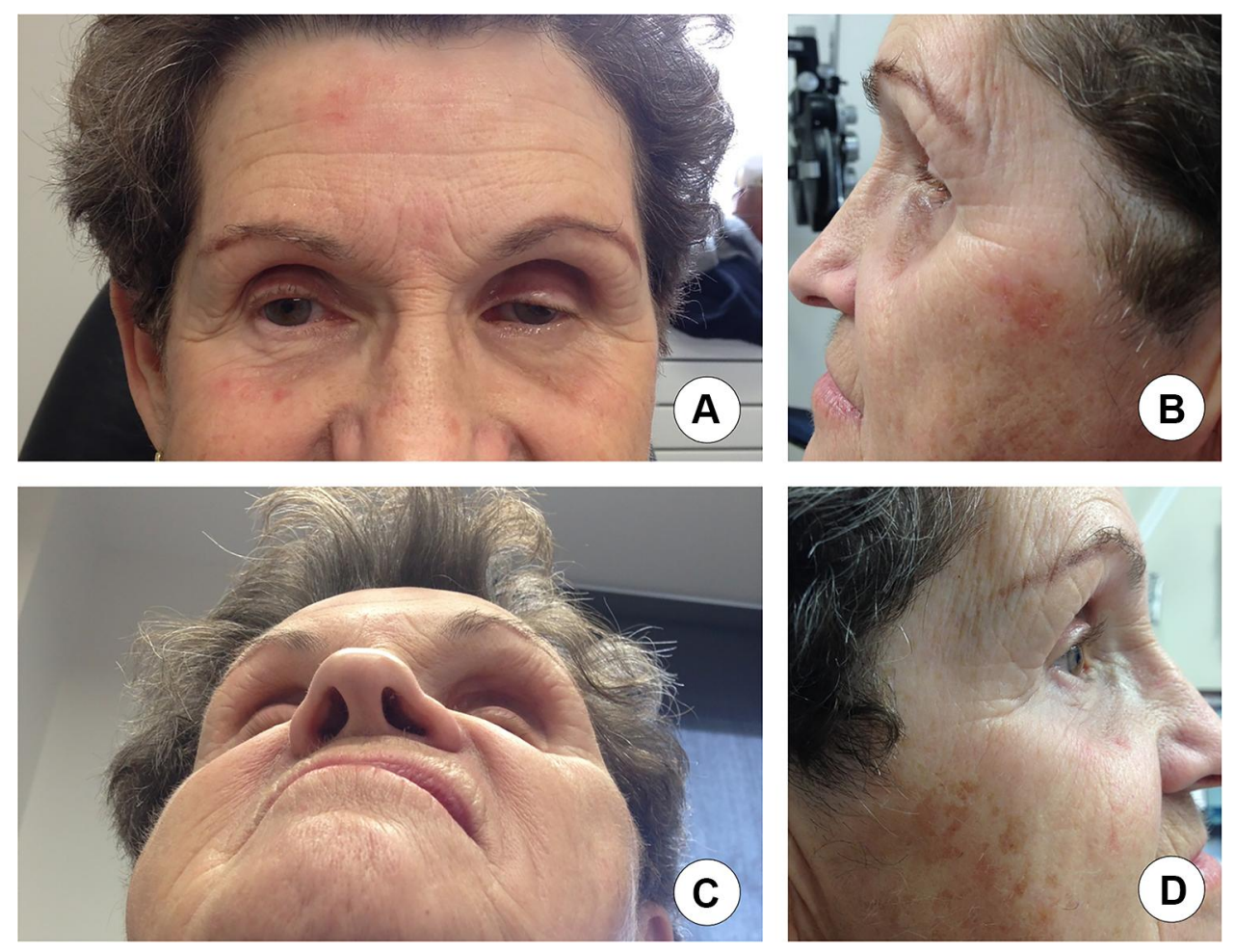

Figure I 74-year old patient with left upper eyelid ptosis and enophthalmos (case I). (A) Frontal view showing left ptosis. (B) Left lateral view showing significant enophthalmos of left eye, compared to lateral view of right eye (D). (C) Worm's eye view showing left enophthalmos. 

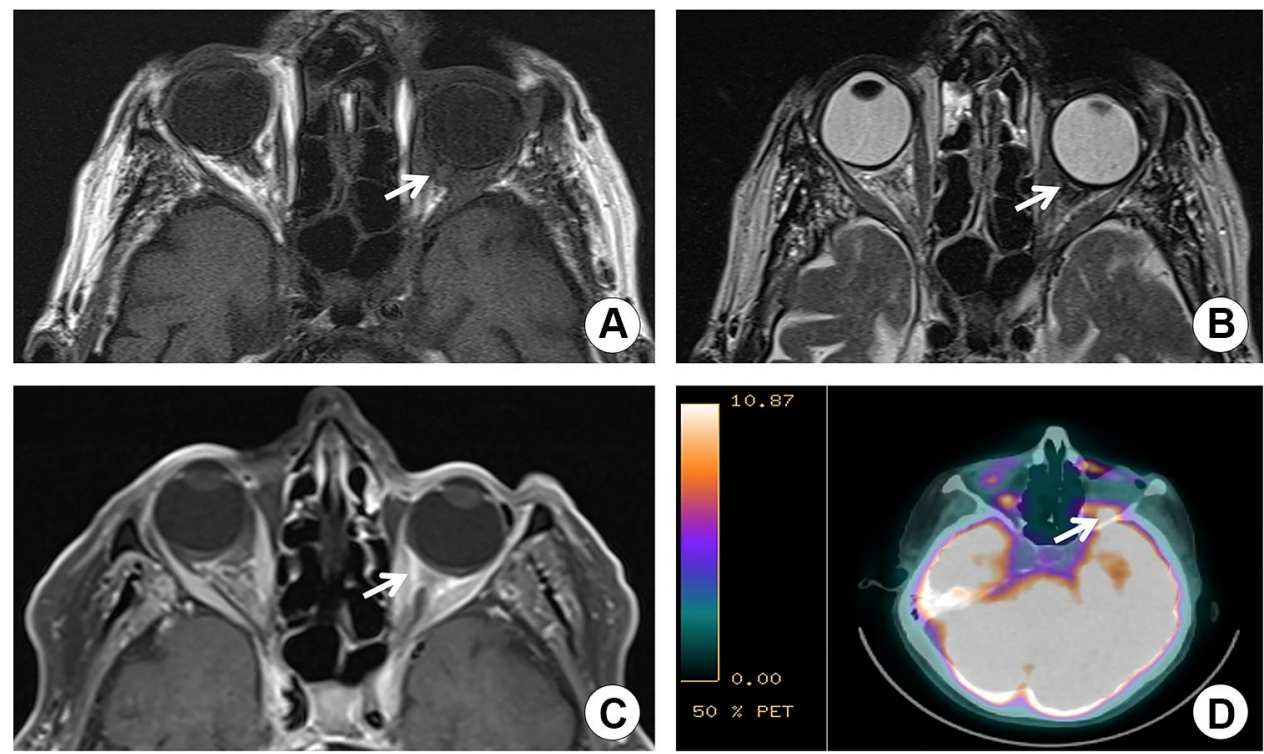

Figure 2 Cerebral and orbital imaging (case I). (A) Axial TI-weighted MRI: lesion (iso-intense to the extraocular muscles) sheathing the intraconal left optic nerve (arrow) with fat atrophy. (B) Axial T2-weighted MRI: hypointense lesion of the left optic nerve (arrow) with enophthalmos. (C) Axial TI-weighted MRI with gadolinium enhancement and fat suppression: enhancing lesion sheathing the left optic nerve (arrow). (D) Cerebral PET-scan: hypermetabolic left inferoposterior orbital lesion (arrow).

Abbreviations: MRI, magnetic resonance imaging; PET, positron emission tomography.

fat atrophy; a diagnosis of breast orbital metastasis was suspected in the differential (Figure 2A-C). Thorough breast imaging studies (mammography, ultrasound and MRI) showed a BIRADS (Breast Imaging Reporting and Data System) IVa lesion described as benign glandular asymmetry, but breast biopsy was not able to confirm any malignancy. The patient underwent a left transcutaneous anterior orbitotomy (TCAO) under local anesthesia for tissue biopsy. The histology and immunohistochemistry (IHC) analyses respectively showed colony-forming units of isolated cells consistent with hormone-receptor-positive and HER2negative metastatic infiltrative breast carcinoma (Figure 3). The patient was started on letrozole by her oncologist.

One year later, although the patient reported no systemic or ocular symptoms besides epiphora OS, a positron emission tomography (PET)-scan revealed multiple bone metastases in addition to progression of the left orbital tumor, mostly located in the inferoposterior orbital region (Figure 2D). The bone biopsy was compatible with infiltrative breast carcinoma metastasis. Breast imaging was repeated, displaying the left breast benign glandular asymmetry which had now evolved to BIRADS IVc, but once again, the breast biopsy was inconclusive. Denosumab and palbociclib were also added to the treatment regimen.

Six years after initial presentation, the patient remains on a dual therapy of letrozole and palbociclib. Although her bone metastases progressed over time, the orbital involvement remained stable, with preserved visual function.

\section{Case 2}

A 76-year-old woman consulted for progressive enophthalmos OD with ipsilateral ptosis. She denied any history of trauma involving the orbital region. Her past medical history was negative for neoplasia. She was known for type 2 diabetes and proliferative diabetic retinopathy OD.

The ophthalmologic examination confirmed right ptosis and enophthalmos associated with resistance to globe retropulsion (Figure 4). An induration of the ipsilateral lacrimal gland was also noticed. There were subtle extraocular motility limitations in both eyes $(-1$ elevation OD; -1 adduction OS). Pupils were equal and reactive, with no afferent pupillary defect, and colour vision was normal (HRR testing score of 10/10).

A cerebral CT-scan with contrast revealed an osteolytic lesion of the right frontal bone, as well as infiltrative lesions in both orbits (extraconal fat OD, lacrimal gland OD, superior rectus muscle OD, medial and inferior rectus muscles OS, and optic nerve OS), presumably secondary to a neoplastic process. The patient underwent a right TCAO with tumor biopsy, which concluded on a diagnosis of breast carcinoma metastasis (lobular 

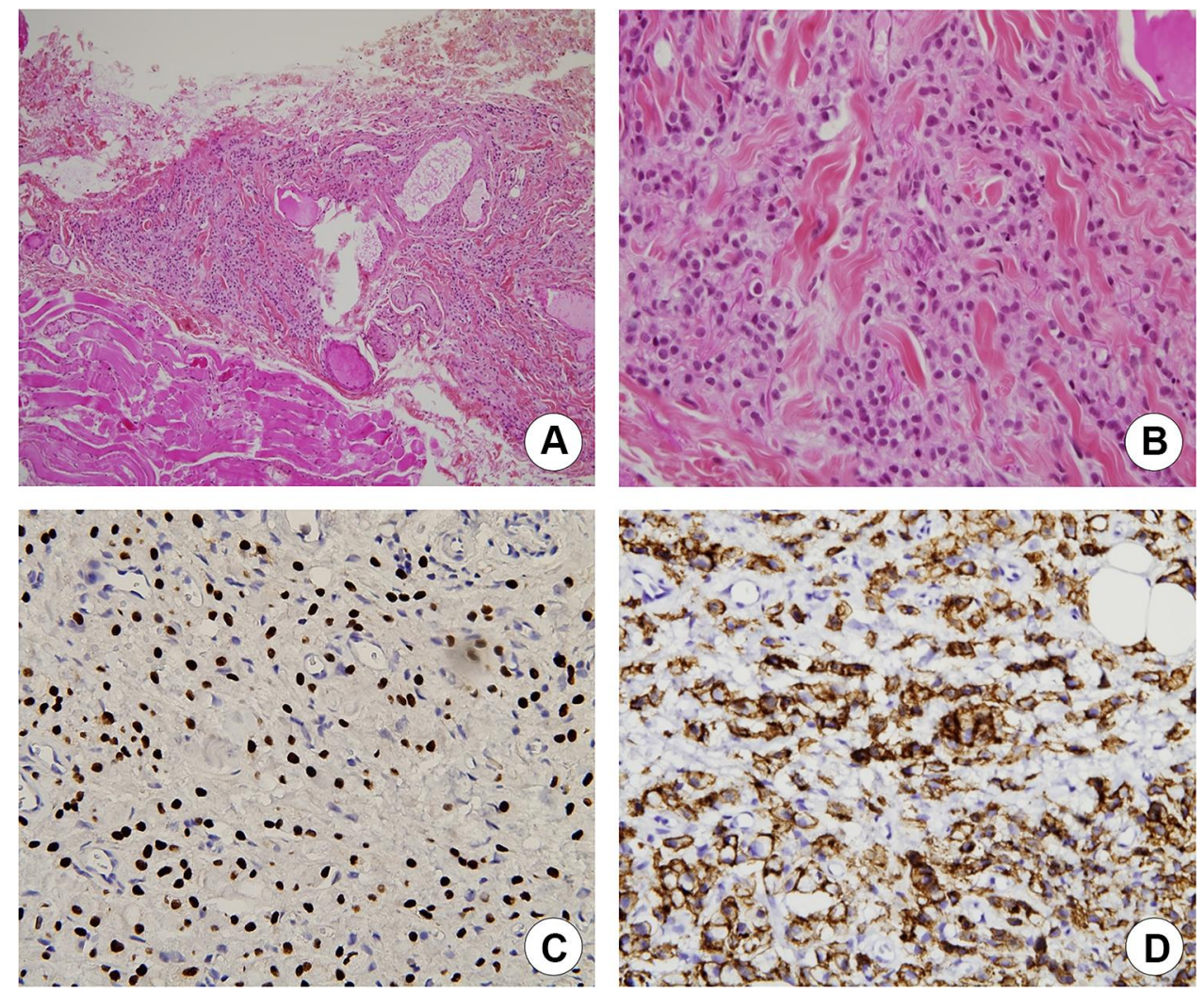

Figure 3 Histopathologic examination of left orbital biopsy specimen (case I). (A) Section of biopsy specimen showing neoplastic cells (HPS stain, $\times 10)$. (B) Increased nuclear size with increased nuclear/cytoplasmic ratio. Infiltration of normal muscle fibers and adipocytes by neoplastic cells (HPS stain, $\times 40$ ). (C) Immunohistochemical staining of tumor cells, suggesting a metastatic breast adenocarcinoma (immunohistochemical staining positive for GATA3, $\times 200$ ). (D) Immunohistochemical staining of tumor cells, suggesting a metastatic breast adenocarcinoma (immunohistochemical staining positive for E-cadherin, $\times 200$ ).

Abbreviation: HPS, hematoxylin-phloxine-saffron.
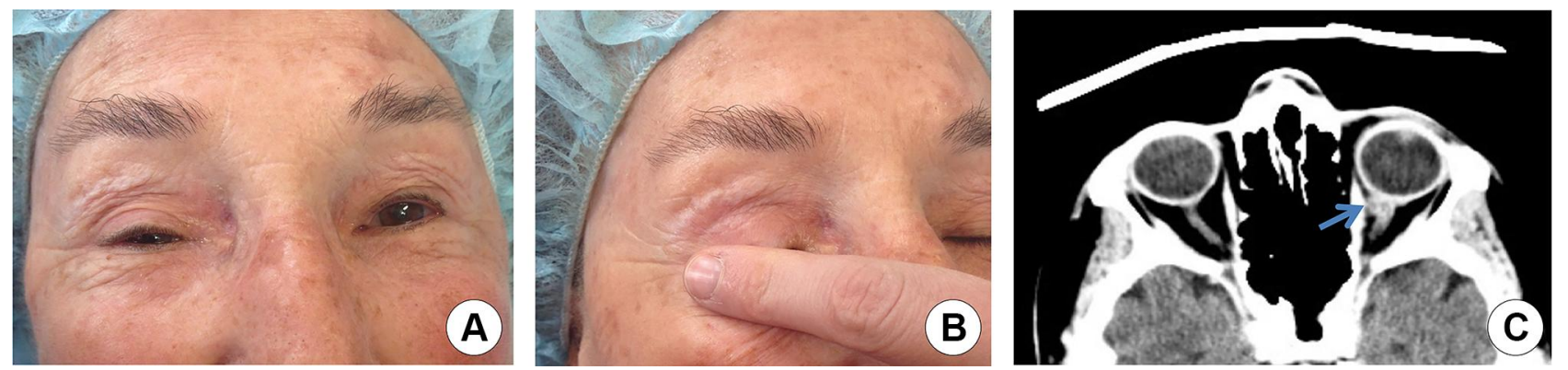

Figure 4 76-year old patient with right ptosis and enophthalmos (case 2). (A) Frontal view showing right ptosis. (B) Frontal view showing an area of subcutaneous tissue induration in front of the lacrimal gland. (C) Axial cerebral CT-scan with contrast showing enhancing metastatic lesion (arrow) adjacent to the optic nerve, at the level of the medial rectus muscle.

Abbreviation: $\mathrm{CT}$, computed tomography.

subtype). IHC analyses detected highly positive hormone receptors and negative HER-2 (Figure 5). Complete physical examination only revealed a left axillary adenopathy; no palpable breast mass was found. A PET-scan revealed a hypermetabolic region in the supero-outer quadrant of the patient's left breast, as well as multiple hypermetabolic lymph nodes implicating the left axillary region, the mediastinum, and both pulmonary hila. Numerous osteolytic lesions on axial skeleton were also identified.

A biopsy of the left breast mass and left axillary adenopathy confirmed a lobular breast carcinoma graded $2 / 3$, positive for estrogen receptors and negative for progesterone receptors and HER-2. She was started on letrozole, palbociclib, and pamidronate. 

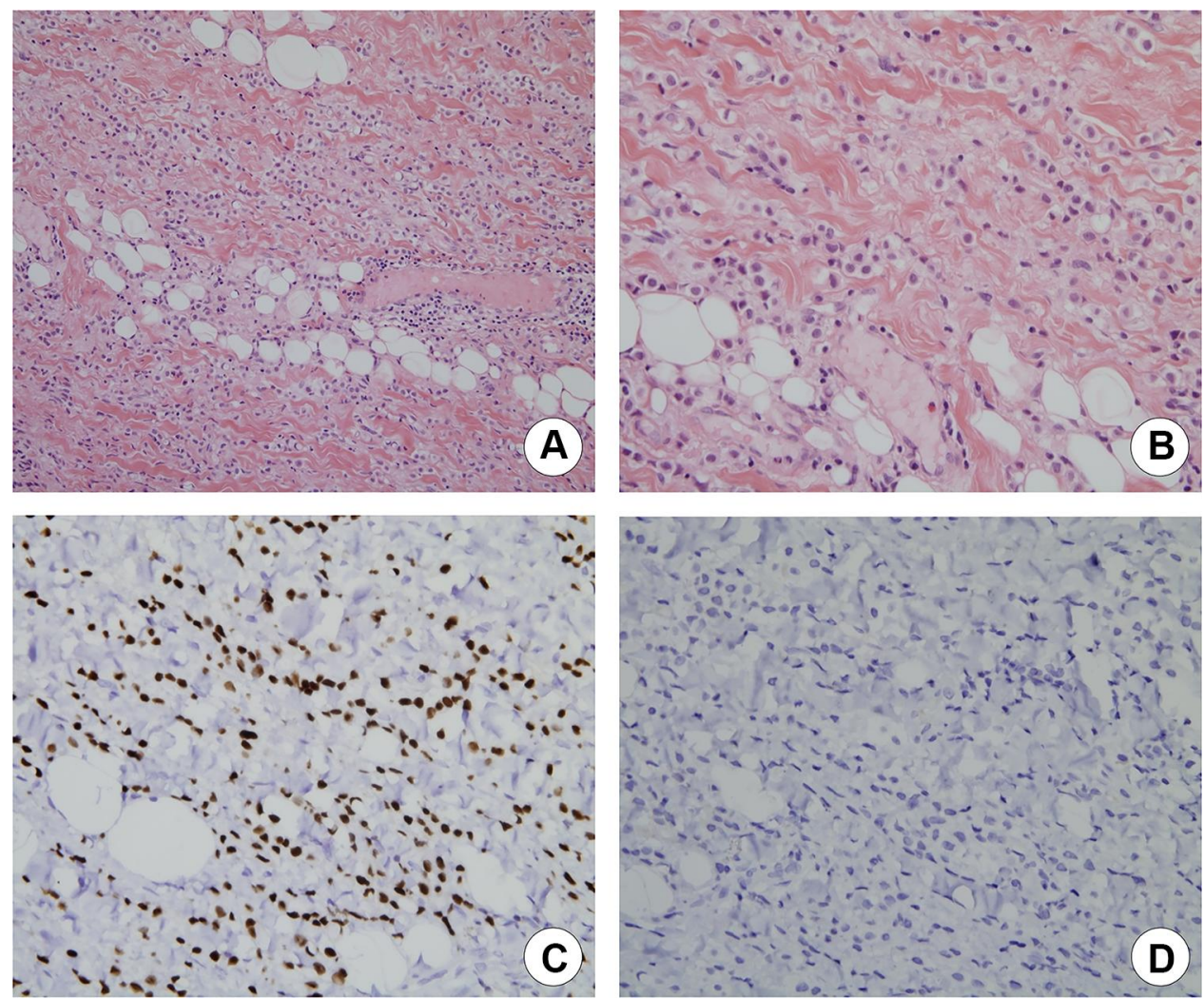

Figure 5 Histopathologic examination of right orbital biopsy specimen (case 2). (A) Section of biopsy specimen showing neoplastic cells. Increased pleomorphism. (H\&E stain, $\times 20$ ). (B) Increased nuclear size with increased nuclear/cytoplasmic ratio. Infiltration of normal muscle fibers and adipocytes by neoplastic cells (H\&E stain, $\times 40$ ). (C) Immunohistochemical staining of tumor cells, suggesting a metastatic adenocarcinoma from breast cancer (immunohistochemical staining positive for GATA3, $\times 200$ ). (D) Immunohistochemical staining of tumor cells, suggesting a metastatic adenocarcinoma from breast cancer, lobular subtype (immunohistochemical staining negative for E-cadherin, $\times 200)$.

Abbreviation: H\&E, hematoxylin and eosin.

A few months later, control imaging revealed a progression of her metastases at the level of the medial rectus muscle OS, as well as presence of a new left frontal lobe lesion with concomitant vasogenic edema. This new lesion was treated with stereotactic radiosurgery $(1800$ centigray $[\mathrm{cGy}]$ radiation dose) and dexamethasone.

Almost three years after initial presentation, the patient died due to progression of her plurimetastatic disease.

\section{Discussion}

Non-traumatic enophthalmos is not always obvious and can be easily unnoticed early on in the course of an insidious disease process. It can present with various signs and symptoms. It would seem that approximately $50 \%$ of patients with enophthalmos are initially referred for investigation of possible exophthalmos of the contralateral eye, ptosis or diplopia. ${ }^{1,4}$ Although non-traumatic enophthalmos has a wide variety of causes, its differential diagnosis includes orbital metastases, and therefore must be investigated thoroughly.
While the breast (48-53\%), prostate gland (12\%), lung $(8 \%)$ and skin (melanoma) $(6 \%)$ are the most frequent sources for orbital metastases, enophthalmos is seen almost exclusively with metastatic scirrhous breast carcinoma. $^{3,5}$

A history of cancer is usually reported before the patient develops any eye concerns. In only a small minority of cases (approximately 19\%), as seen with our two patients, will the OMs arise before the diagnosis of the primary cancer. ${ }^{3,8}$ The average interval between the diagnosis of primary neoplasia and the development of orbital metastases usually varies from 4 to 6 years, but delays of over 20 years have been reported. ${ }^{2,6}$ Age is also an important risk factor for OMs development, as they occur more frequently during or after the sixth decade of life, ${ }^{5,8}$ which was the case in our 2 patients.

Orbital metastases are more often unilateral than bilateral; bilateral orbital metastases are seen most commonly in breast carcinoma ( $20 \%$ of cases), in comparison to other origins (4\% of cases). ${ }^{10}$ Typical manifestations of OMs 
include exophthalmos (most common), pain, diplopia, chemosis and eyelid swelling. In contrast, enophthalmos is seen in less than a quarter of cases and almost exclusively with metastatic breast carcinoma. ${ }^{3}$ The hypothesis is that tumor-induced desmoplasia, fibrosis, muscle invasion and/ or fat atrophy can be responsible for the ocular globe inward traction. ${ }^{1,9}$ While tumors can be located on the walls of the orbit (osseous invasion) as well as on the orbital content, the predilection areas are orbital fat and extraocular muscles. ${ }^{8,10,11}$ Therefore, a progressive nontraumatic enophthalmos associated with restriction of ocular motility is highly alarming for metastatic breast carcinoma, even more so if the patient has bilateral enophthalmos in this setting. ${ }^{1,10}$

When evaluating orbital masses, although MRI has become an essential tool, the gold standard for diagnosis is excisional biopsy. For breast carcinoma, the histologic features vary depending on series. From a meta-analysis of 68 previously published case reports of OMs, invasive lobular breast cancer (ILBC) was the most common malignancy in patients with OMs, and this subtype appeared five times more often than the invasive ductal subtype. ${ }^{6}$ ILBC is a slowly progressive tumor and accounts for $10-15 \%$ of all mammary carcinomas. ${ }^{6}$ Its growth is driven by estrogen stimulation, which explains the high prevalence of positive estrogen receptors (ER) on biopsy specimens and the choice of letrozole and palbociclib hormonotherapy, as in our two patients. ${ }^{6}$ Nonetheless, histologic features and IHC testing of OMs may sometimes also differ from the primary tumor itself. ${ }^{12}$

Orbital metastases often reflect a widespread haematogenous dissemination with frequent plurimetastatic involvement, as shown in our two cases which showed bone and brain metastatic lesions. The overall prognosis is also poor; Font et al found a mean survival time after diagnosis of OMs, regardless of the type of primary cancer, of approximately 15.6 months. ${ }^{13}$ This is however far less than what we report in our 2 patients: the patient from the first case is still alive six years after initial presentation, and the patient from the second case survived for 33 months after initial presentation. The treatment of OMs is usually palliative and aims to improve patients' quality of life and to restore or preserve their vision, although good outcomes with aggressive treatment has been reported. ${ }^{12-16}$ Besides hormone therapy, radiotherapy and chemotherapy are other treatment options that are increasingly evolving. In general, extensive orbital surgery to remove the metastasis is not recommended since the risk of associated morbidity is high, and it is not curative; enucleation seems to show no survival benefit and should be reserved for specific cases such as refractory ocular pain or unmanageable hygiene due to a rapidly growing tumor. ${ }^{12-16}$

\section{Conclusion}

Although rare, non-traumatic enophthalmos has a wide differential diagnosis and can be the sole presenting feature of an undiagnosed metastatic disease. A thorough history, complete physical examination, comprehensive eye exam by a specialist, orbital and systemic imaging studies, biopsy of a suspicious lesion and timely referral to an oncology department for a multidisciplinary evaluation and management are essential tools to get a definitive diagnosis and plan the appropriate treatment. In the upcoming years, development of novel targeted agents and radiotherapy techniques will hopefully improve quality of life, help preserve ocular function and increase life expectancy of these patients. To conclude, this case series serves as a strong reminder that enophthalmos can be the presenting feature of a patient with metastatic breast carcinoma, in absence of systemic symptoms, and with no prior history of neoplasia.

\section{Summary}

Although rare, non-traumatic enophthalmos has a wide differential diagnosis and can be the sole presenting feature of an undiagnosed metastatic disease. The authors report the cases of two women who developed enophthalmos as the first clinical manifestation of a previously unsuspected metastatic breast carcinoma.

\section{Consent}

This case series does contain identifying details concerning the patient; however, in compliance with The Committee on Publication Ethics (COPE) guidelines, written consent to publish personal information, pictures and case details has been obtained from the patients. Institutional approval is not necessary to publish the case details according to the author's institution guidelines.

\section{Acknowledgments}

All authors attest that they meet the current ICMJE criteria for Authorship. This article is the authors' original work. It has not been published before, and is not under consideration for publication elsewhere. 


\section{Author Contributions}

All authors contributed to data analysis, drafting or revising the article, have agreed on the journal to which the article will be submitted, gave final approval of the version to be published, and agree to be accountable for all aspects of the work.

\section{Funding}

No funding or grant support was received for this study.

\section{Disclosure}

None of the authors have financial or proprietary interests to disclose.

\section{References}

1. Athanasiov PA, Prabhakaran VC, Selva D. Non-traumatic enophthalmos: a review. Acta Ophthalmol. 2008;86:356-364. doi:10.1111/ j.1755-3768.2007.01152.x

2. Gonçalves AC, Moura FC, Monteiro ML. Bilateral progressive enophthalmos as the presenting sign of metastatic breast carcinoma. Ophthal Plast Reconstruct Surg. 2005;21:311-313. doi:10.1097/01. iop.0000167786.00697.0b

3. Shields JA, Shields CL, Brotman HK, et al. Cancer metastatic to the orbit: the 2000 Robert M. Curts Lecture. Ophthal Plast Reconstruct Surg. 2001;17:346-354. doi:10.1097/00002341-200109000-00009

4. Cline RA, Rootman J. Enophthalmos: a clinical review. Ophthalmology. 1984;91:229-237. doi:10.1016/S0161-6420(84)34299-3

5. Shields JA, Shields CL, Scartozzi R. Survey of 1264 patients with orbital tumors and simulating lesions: the 2002 Montgomery Lecture, part 1. Ophthalmology. 2004;111:997-1008. doi:10.1016/j. ophtha.2003.01.002

6. Raap M, Antonopoulos W, Dämmrich M, et al. High frequency of lobular breast cancer in distant metastases to the orbit. Cancer Med. 2015;4:104-111. doi:10.1002/cam4.331
7. Holland D, Maune S, Kovács G, Behrendt S. Metastatic tumors of the orbit: a retrospective study. Orbit. 2003;22:15-24. doi:10.1076/ orbi.22.1.15.14007

8. Cornelis F, Mejdoubi M, Dousset V. Bilateral orbital extension of breast cancer metastasis. $J$ Radiol. 2007;88:684-686. doi:10.1016/ S0221-0363(07)89876-3

9. Lagreze WD, Wesendahl TA, Kommerell G. Enophthalmos caused by orbital metastasis of breast carcinoma. Klin Monatsbl Augenheilkd. 1997;211:68-69.

10. Goldberg RA, Rootman J. Clinical characteristics of metastatic orbital tumors. Ophthalmology. 1990;97:620-624. doi:10.1016/S01616420(90)32534-4

11. Tomizawa Y, Ocque R, Ohori NP. Orbital metastasis as the initial presentation of invasive lobular carcinoma of breast. Intern Med. 2012;51:1635-1638. doi:10.2169/internalmedicine.51.7641

12. Eckardt AM, Rana M, Essig H, Gellrich NC. Orbital metastases as first sign of metastatic spread in breast cancer: case report and review of the literature. Head Neck Oncol. 2011;3:37. doi:10.1186/17583284-3-37

13. Font RL, Ferry AP. Carcinoma metastatic to the eye and orbit III. A clinicopathologic study of 28 cases metastatic to the orbit. Cancer. 1976;38:1326-1335.

14. Char DH, Miller T, Kroll S. Orbital metastases: diagnosis and course. Br J Ophthalmol. 1997;81:386-390. doi:10.1136/bjo.81.5.386

15. Mehdi Ahmad S, Esmaeli B. Metastatic tumors of the orbit and ocular adnexa. Curr Opin Ophthalmol. 2007;18:405-413. doi:10.1097/ICU.0b013e3282c5077c

16. Mohadjer Y, Holds JB. Orbital metastasis as the initial finding of breast carcinoma: a ten-year survival. Ophthal Plast Reconstruct Surg. 2005;21:65-66. doi:10.1097/01.IOP.0000150350.35376.11
International Medical Case Reports Journal

\section{Publish your work in this journal}

The International Medical Case Reports Journal is an international, peer-reviewed open-access journal publishing original case reports from all medical specialties. Previously unpublished medical posters are also accepted relating to any area of clinical or preclinical science. Submissions should not normally exceed 2,000 words or 4

\section{Dovepress}

published pages including figures, diagrams and references. The manuscript management system is completely online and includes a very quick and fair peer-review system, which is all easy to use. Visit http://www.dovepress.com/testimonials.php to read real quotes from published authors. 